\title{
Indian Pediatrics Case Reports (IPCaRes) Launching a New Journal from the Indian Academy of Pediatrics
}

Clinical cases are the first step of the medical student into the world of clinical medicine. Discussion on a clinical case gives the student - or for that matter, any clinician, a well-rounded view of the patient's problems, the likely causation, and the management and outcome. By extension, published case reports provide the readers exposure to the events at the frontline of clinical medicine, whether it is some rare manifestation or complication, an innovative diagnostic or therapeutic approach, or a novel association, like the one which lead to the initial identification of acquired immune deficiency syndrome. Moreover, for the practicing pediatrician, away from the esoteric concepts of epidemiology and research, it is the case-report section that provides a sense of belonging to a scientific journal. This is more relevant for journals like ours that are published by professional bodies. On the other hand, for a young doctor foraying into academic pediatrics, it is the case report section that provides both an avenue to get your feet wet in the field of publication and also an outlet for demonstrating your scientific writing skills.

Although the first written case description may be more than a few centuries old; in recent years, most journals have been reluctant to publish case reports. Even those that are publishing, continue to restrain them by limiting the word count and the number of authors. Moreover, the rejection rate for this section is one of the highest for most journals, reaching 95\% for Indian Pediatrics. The reasons are multifold, including a gross disparity between the space we have and the number of submissions we receive.

Looking at the multi-faceted advantages of case reports and the need to make available this academic avenue to the majority of our academy members, we propose to start a new journal exclusively dedicated to publishing case reports, Indian Pediatrics Case Reports (IP CaRes). This journal will be a quarterly journal publishing case reports, case series, images, clinical videos, correspondence and related information, with ecopy mailed free to all members of the Indian Academy of Pediatrics. Print issues will be available for subscription to individuals and libraries. This high volume journal shall publish cases related to all pediatric subspecialties and allied specialties. There would not be any restriction on the number of authors, provided they meet authorship criteria, and word limits will be not too restrictive. The editorial board has already been constituted, author guidelines prepared, and the website shall be up and running within next few months. The journal will share offices with Indian Pediatrics.

We plan to publish the first issue in early 2021, and submissions are welcome at IPCaRes2020@gmail.com.

\section{Devendra Mishra ${ }^{1 *}$ And Piyush Gupta ${ }^{2}$ ${ }^{1}$ Editor-in-Chief, Indian Pediatrics, and ${ }^{2}$ President-Elect 2020, Indian Academy of Pediatrics. *ip.editor@iapindia.org}

